\title{
DEVELOPMENT OF A MICROPROCESSOR-CONTROLLED COULOMETRIC SYSTEM FOR STABLE pH CONTROL
}

\author{
P. BERGVELD,* B. H. v.d. SCHOOT and J. H. L. ONOKIEWICZ
}

Department of Electrical Engineering, Twente University of Technology, P.O. Box 217, 7500 AE Enschede (The Netherlands)

(Received 17th January 1983)

\section{SUMMARY}

The coulometric pH control system utilizes a programmable coulostat for controlling the $\mathrm{pH}$ of a certain volume of unbuffered solution. Based on theoretical considerations, conditions are established which guarantee stable operation with maximum suppression of disturbances from the dissolution of carbon dioxide, for example. It is shown that the dynamic properties of the control system depend greatly on the response time of the $\mathrm{pH}$ sensor which measures the actual $\mathrm{pH}$. The best results are therefore obtained by using an ISFET as pH sensor.

The determination of variations in offset and sensitivity of glass $\mathrm{pH}$ electrodes and other $\mathrm{pH}$ sensors such as ISFET's, necessitates the availability of a certain volume of an aqueous solution, the $\mathrm{pH}$ of which can be controlled over a long period and is not affected by external interferences, e.g., by dissolution of carbon dioxide. To keep the $\mathrm{pH}$ of a solution constant, a buffer is mostly used. However, drift in the output voltage of a $\mathrm{pH}$ sensor can be detected in this way, only as a function of available buffer solutions which are used in succession, while the sensors have to be cleaned thoroughly between measurements. Any initial drifts observed cannot be interpreted in this way, because they depend more on the cleaning procedure than on the previous $\mathrm{pH}$ value. A procedure of successively dipping in different buffers with cleaning procedures in between is also unsuitable for the determination of variations in the sensitivity and the response time of $\mathrm{pH}$ sensors.

A more appropriate procedure is the addition of acid or base to change the $\mathrm{pH}$ of a solution and to control the required $\mathrm{pH}$, which may be constant or variable, by means of a closed-loop control system. In this case, the actual $\mathrm{pH}$ of the test solution is continuously measured and compared with a preprogrammed reference pattern. The difference between the measured $\mathrm{pH}$ and the required $\mathrm{pH}$ is automatically kept at a minimum, e.g., by means of a set of controllable motor burettes which add the correct amount of acid or base. A disadvantage of such a system is that the volume of the test solution constantly increases and thus also the ionic strength, which may affect the response of the sensors under test. A second disadvantage may be a limitation 
of the quantity of acid or base to be added, thus restricting the time of a continuous test.

To eliminate such problems, a $\mathrm{pH}$ control system was designed in which $\mathrm{pH}$ changes are produced coulometrically and where a microprocessor is applied to control a coulostat. The desired flexibility is obtained by the choice of software in relation to the operational design of the whole system. A block diagram of this system is shown in Fig. 1. Stable and rapid operation of the system requires that the design complies with certain rules. These can be established by applying known control engineering criteria in relation to certain properties of the different blocks given in Fig. 1. Accordingly, these blocks will first be discussed separately.

\section{DESCRIPTION OF THE SYSTEM}

\section{The test set-up}

The $\mathrm{pH}$ of the test solution is changed by coulometric action, in which an electric current generates $\mathrm{H}^{+}$or $\mathrm{OH}^{-}$ions at a platinum electrode in accordance with the chemical reactions:

$$
\begin{aligned}
& 2 \mathrm{H}_{2} \mathrm{O} \rightarrow 4 \mathrm{H}^{+}+\mathrm{O}_{2}+4 \mathrm{e}^{-} \\
& 2 \mathrm{e}^{-}+2 \mathrm{H}_{2} \mathrm{O} \rightarrow \mathrm{H}_{2}+2 \mathrm{OH}^{-}
\end{aligned}
$$

at the anode and cathode, respectively. In order to avoid other reactions than those given in Eqns. (1) and (2) for a broad $\mathrm{pH}$ range, the test solution chosen was $1 \mathrm{M}$ sodium sulphate or $1 \mathrm{M}$ sodium perchlorate $(50 \mathrm{ml})$. In order to avoid neutralization of generated $\mathrm{H}^{+}$and $\mathrm{OH}^{-}$ions, the test solution in which the platinum gauze working electrode was inserted, was connected
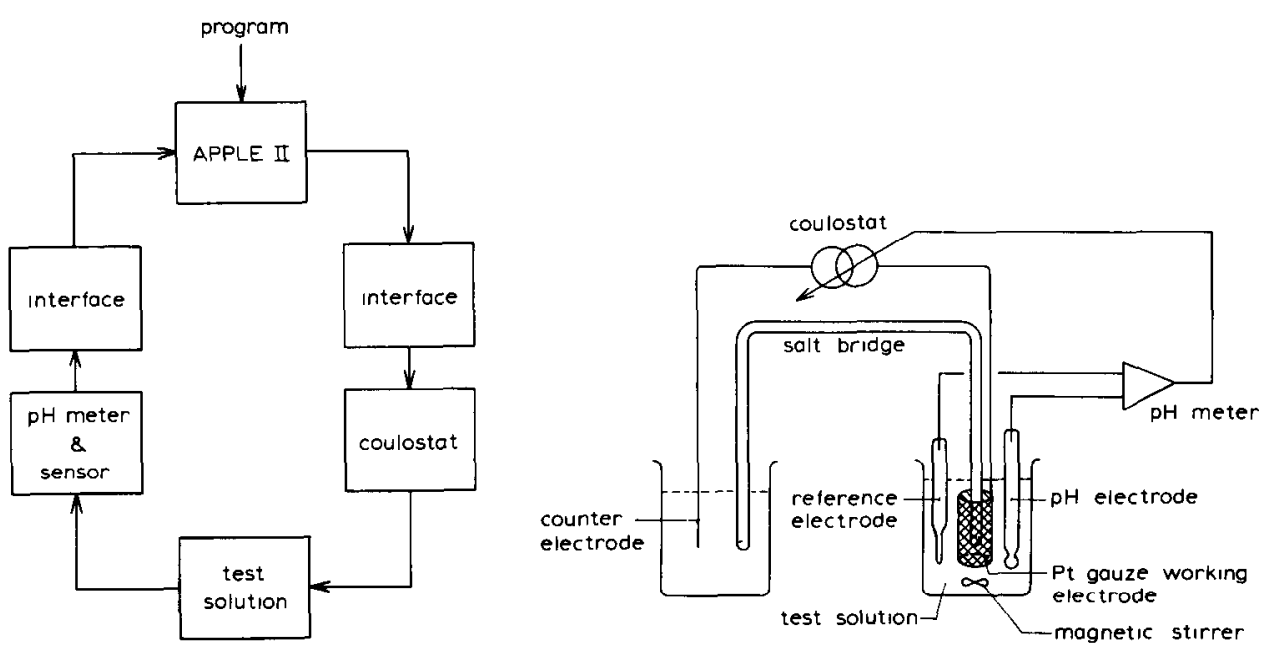

Fig. 1. Block diagram of the $\mathrm{pH}$ control system.

Fig. 2. Experimental set-up of the coulometric control system. 
by a salt bridge (containing agar-agar and sodium perchlorate) to a second solution of the same composition provided with a platinum counter electrode. The test solution also contained a $\mathrm{pH}$-measuring electrode and a reference electrode to record the generated $\mathrm{pH}$, as shown in Fig. 2.

To avoid any direct influence of the coulometric current on the $\mathrm{pH}$ measurement, the generating electrode in the test solution was a platinum gauze cylinder around the salt bridge contact. Outside this working electrode, the electric field was almost negligible. Further, any effect of a small stray field could be minimized by proper positioning of the $\mathrm{pH}$ sensor with respect to its reference electrode. The test solution was thermostated at the required temperature.

\section{The coulostat}

The current source used was a Metrohm E524 coulostat, which can deliver preprogrammed currents $(I)$ at floating electrodes during a certain time $T$. The output is expressed in moles of $\mathrm{H}^{+}$or $\mathrm{OH}^{-}$ions: for $I>0$, the moles of $\mathrm{H}^{+}$generated is $\int_{0}^{T}(I \mathrm{~d} t / F)$ and for $I<0$, the moles of $\mathrm{OH}^{-}$generated is $\int_{0}^{T}(-I \mathrm{~d} t / F)$, where $F$ is the Faraday constant $\left(F=96487 \mathrm{C} \mathrm{mol}^{-1}\right)$. The corresponding transfer function in $\mathrm{pH} \mathrm{mol}^{-1}$ can be described as $H_{c}(s)=K_{1} / s F$, where $s$ is the Laplace variable and $K_{1}$ is a function of the volume of the test solution, its composition, and the actual $\mathrm{pH}$, caused by the non-linearity of the titration curve.

To keep the current source floating, the coulostat is controlled at its appropriate control input by means of two opto-couplers which are activated by the microprocessor. Because the electronic circuitry for the implementation of opto-couplers is much simpler for digital than for analog application, the required output from the coulostat is obtained by a preprogrammed constant value of the output current during a certain time $T$ which is determined by the microprocessor and corresponds with the desired control program. The direction of the current is also controlled by the microprocessor, by activating one opto-coupler for a positive current and the other for a negative current.

\section{The pH measurement}

The actual control of the $\mathrm{pH}$ of the test solution is achieved by measuring and comparing it with the required value. Obviously, this measurement of the $\mathrm{pH}$ determines the accuracy of the $\mathrm{pH}$ control. Any type of $\mathrm{pH}$ sensor can be used for this measurement, although they should in principle be better than the $\mathrm{pH}$ sensors which have to be tested by means of programmed $\mathrm{pH}$ variations of the test solution. This aspect will receive more attention below, in considering the response time of the system.

In general, any $\mathrm{pH}$ sensor and its attached amplifier will respond to a variation in the $\mathrm{pH}$ with a time constant $\tau_{e}$. This time constant may be a function of the actual $\mathrm{pH}$ of the test solution. For instance, it is known that a glass $\mathrm{pH}$ electrode is rather slow around $\mathrm{pH} 7$ but faster at higher and lower 
$\mathrm{pH}$ values. The transfer function for such an electrode can be written as

$H_{e}(s)=1 /\left(1+s \tau_{e}\right)$

Of course, the $\mathrm{H}^{+}$or $\mathrm{OH}^{-}$ions generated at the gauze electrode near the $\mathrm{pH}$ sensor are not immediately available at the place of measurement. Even when the solution is well stirred, there will always be a certain transportation lag between the working electrode and the sensor, with a corresponding delay time constant $\tau_{d}$, as well as simultaneously occurring mixing, introducing another time constant $\tau_{m}$. Both effects result in a transfer function in the system, which can be described as

$H_{d}(s)=\left[\exp \left(-s \tau_{d}\right)\right] /\left(1+s \tau_{m}\right)$

\section{The microprocessor interfaces}

As already mentioned, the interface between the microprocessor and the coulostat merely provides a digital signal applied to one of two optocouplers. The interface with the $\mathrm{pH}$ meter consists of an $\mathrm{A} / \mathrm{D}$ converter which samples the analog $\mathrm{pH}$ signal with a sampling frequency $1 / T_{s}$. This introduces an additional transfer function

$H_{s}(s)=\left[1-\exp \left(-s T_{s}\right)\right]\left[1 / s T_{s}\right]$

which can be approximated for low frequencies by

$H_{s}(s)=\exp \left(-s T_{s} / 2\right)$

Thus this sampling introduces a delay of $T_{s} / 2$ seconds.

\section{The complete control loop}

The separate descriptions of the various blocks given above allow the overall system to be represented as shown in Fig. 3. In this figure, the transfer functions for sampling, coulostat, transport/mixing and $\mathrm{pH}$ measurement can be distinguished, while two blocks are added, namely a proportional controller, characterized by $K_{2}(A / \mathrm{pH})$ and a block that represents the relation $K_{1} / s$ between an interfering action (e.g., dissolution of carbon dioxide) and the resulting $\mathrm{pH} \mathrm{mol}^{-1}$. This $K_{1}$ is the same factor as is used for the coulostat, and so is a function of the test solution and its $\mathrm{pH}$.

The overall transfer function (closed-loop amplification) of the system is

$$
\begin{aligned}
H_{\text {closed loop }}= & \exp \left[-s\left(\tau_{d}+T_{s} / 2\right)\right] /\left\{\exp \left[-s\left(\tau_{d}+T_{s} / 2\right)\right]\right. \\
& \left.+\left[F s\left(1+s \tau_{e}\right)\left(1+s \tau_{m}\right) / K_{1} K_{2}\right]\right\}
\end{aligned}
$$

which value approaches unity for $s \rightarrow 0$.

The suppression of the interference signal is

$$
\begin{aligned}
H_{\text {interference }}= & \left(F / K_{2}\right)\left(1+s \tau_{m}\right) /\left\{\exp \left[-s\left(\tau_{d}+T_{s} / 2\right)\right]\right. \\
& \left.+\left[F s\left(1+s \tau_{e}\right)\left(1+s \tau_{m}\right) / K_{1} K_{2}\right]\right\}
\end{aligned}
$$

which value approaches $F / K_{2}$ for $s \rightarrow 0$. 


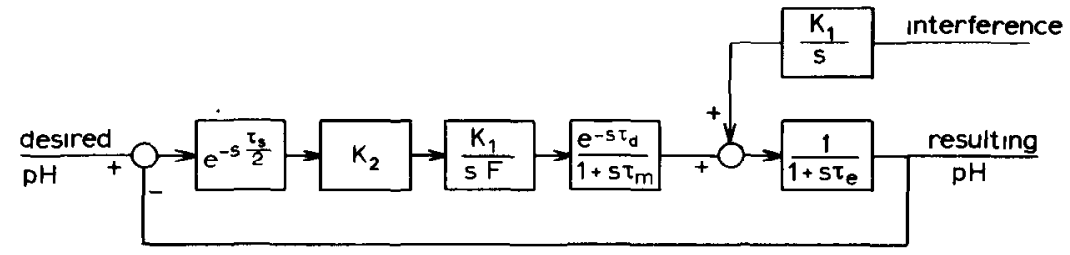

Fig. 3. The pH control loop.

A maximum suppression of interference can thus be expected for a large value of $K$. The value of $K_{2}$, however, is limited by the general control criteria for stable closed-loop systems, which can be determined from the amplitude and the phase diagram (Bode diagrams) [1] of the open-loop amplification as given by

$H_{0}(s)=K_{1} K_{2} \exp \left[-s\left(\tau_{d}+T_{s} / 2\right)\right] /\left[s F\left(1+s \tau_{e}\right)\left(1+s \tau_{m}\right)\right]$

From Eqn. (9) it follows that

$|H(j \omega)|=\left(K_{1} K_{2} / F\right) / \omega\left[\omega^{2}\left(\tau_{e}+\tau_{m}\right)^{2}+\left(1-\omega^{2} \tau_{e} \tau_{m}\right)^{2}\right]^{1 / 2}$

$\phi(j \omega)=-\frac{\pi}{2}-\omega\left(\tau_{d}+\frac{T_{s}}{2}\right)-\operatorname{arctg}\left(\tau_{e} \omega\right)-\operatorname{arctg}\left(\tau_{m} \omega\right)$.

where $\omega$ is the circular frequency $(j \omega=s)$.

The control criteria are as follows: for the phase margin, $|H(j \omega)|=1$ and $\phi\left(j \omega_{1}\right) \leqslant 50^{\circ}$; and for the gain margin, $\phi(j \omega)=-180^{\circ}$ and $\left|H\left(j \omega_{2}\right)\right| \leqslant 0.5$. Both conditions have to be fulfilled. The tolerable value of $K_{2}$ can be calculated from Eqns. (11) and (10) in relation to given values of $\tau_{d}, \tau_{m}, T_{s}$, $\tau_{e}(\mathrm{pH})$, and $K_{1}(\mathrm{pH})$. The optimal control over a broad $\mathrm{pH}$ range requires that the microprocessor contain a preprogrammed table of the $K_{2}$ values, which are previously determined experimentally in the given set-up as described in the next section.

\section{DETERMINATION OF THE ESSENTIAL SYSTEM PARAMETERS}

In order to guarantee stable operation of the closed-loop system described in the previous section, the essential parameters $\tau_{d}, \tau_{m}, \tau_{e}(\mathrm{pH})$ and $K_{1}(\mathrm{pH})$ have to be measured or calculated beforehand from that part of the system that fixes these parameters, i.e., the measuring vessel with the working electrode and the $\mathrm{pH}$ sensor.

To determine the other parameters, a current pulse $I$ is generated for a certain time $T$ in the experimental set-up, and the resulting $\Delta \mathrm{pH}$, which must be small enough for the system to be considered linear, is recorded by the pH sensor. A typical result of such an experiment is shown in Fig. 4.

It was found from these experiments that $\tau_{d}$ is 1.5 seconds. The values of $\tau_{e}$ and $\tau_{m}$ cannot be determined separately because the effects are mixed. 


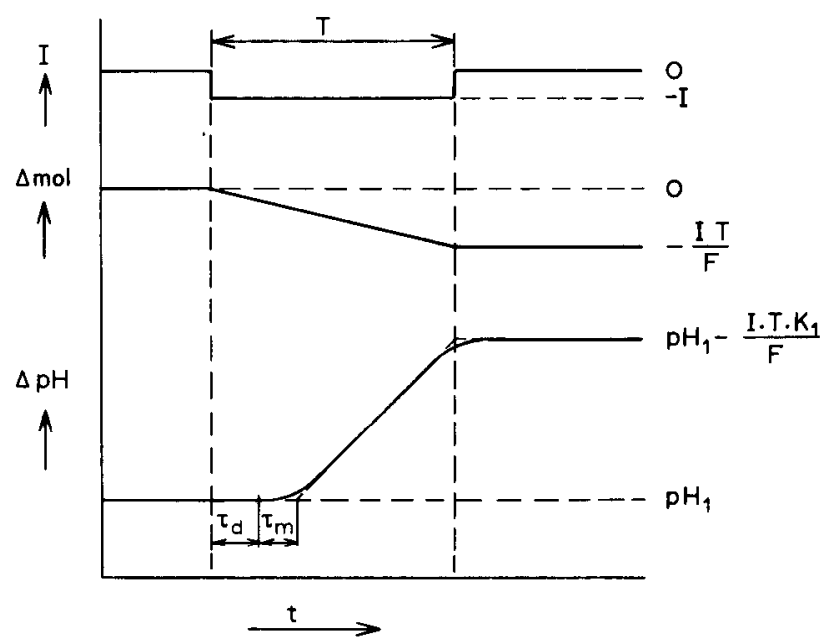

Fig. 4. Experimental result of $\Delta \mathrm{pH}=f(\Delta \mathrm{mol})$, measured with an ISFET.

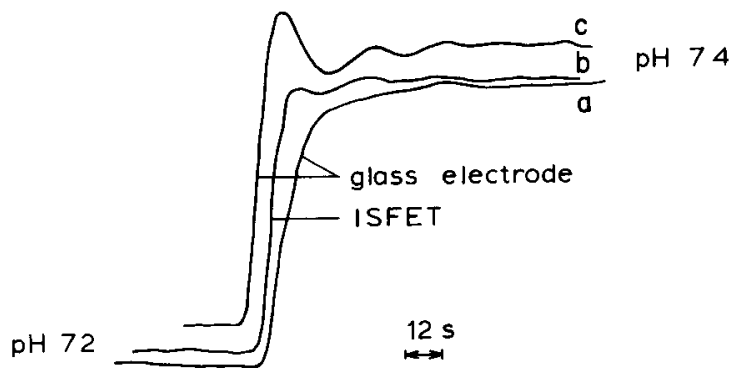

Fig. 5. Programmed stepwise change in the $\mathrm{pH}$ of the test solution. Curves: (a) glass electrode with $K_{2}=7.9 \rightarrow 6.1$; (b) ISFET with $K_{2}=15.8 \rightarrow 12.3$; (c) glass electrode with $K_{2}=$ $15.8 \rightarrow 12.3$.

However, if an ISFET is used as the $\mathrm{pH}$ sensor, the time constant $\tau_{e}$ will be very small (of the order of $\mathrm{ms}$ ) [2], and the value of $\tau_{m}$ can be measured. The value found was 0.5 second. Both time constants, $\tau_{d}$ and $\tau_{m}$, are independent of $\mathrm{pH}$.

When a glass electrode is used as the $\mathrm{pH}$ sensor, the value of $\tau_{e}$ must be considered, but it is not possible to measure this value within the above experiment. In order to be certain, the worst case for the $\tau_{e}$ value of 3 seconds around $\mathrm{pH} \mathrm{7,} \mathrm{known} \mathrm{from} \mathrm{the} \mathrm{literature,} \mathrm{is} \mathrm{taken} \mathrm{into} \mathrm{account} \mathrm{for}$ the whole $\mathrm{pH}$ range.

The values of $K_{1}\left(\mathrm{pH} \mathrm{mol}{ }^{-1}\right)$ as a function of $\mathrm{pH}$ can be determined from a sequence of experiments as described above. The values of $K_{1}$ can also be determined from a titration curve. Table 1 gives the calculated values for $K_{1}$, as well as the corresponding maximum values of $K_{2}$ for a glass electrode and 


\section{TABLE 1}

Measured values of $K_{1}$ as a function of $\mathrm{pH}$ and calculated maximum values of $K_{2}$. The control program uses such a table in the pH range 3-11. The values $K_{2}$ in brackets give the pulse duration in milliseconds when the coulostat is set at $9.65 \mathrm{~mA}\left(0.1 \mu \mathrm{mol} \mathrm{s} \mathrm{s}^{-1}\right)$ for an error of $0.01 \mathrm{pH}$. The values are given for a test volume of $50 \mathrm{ml}$

\begin{tabular}{llll}
\hline $\mathrm{pH}$ & $K_{1}\left(\mathrm{pH} \mu \mathrm{mol}^{-1}\right)$ & $\frac{K_{2}(\mathrm{~mA} / \mathrm{pH})}{n}$ & $\frac{K_{2}(\mathrm{~mA} / \mathrm{pH})}{\text { ISFET }^{\mathrm{b}}}$ \\
\hline 6.00 & & Glass electrode & \\
6.25 & 0.34 & $39.8(41)$ & $80.1(83)$ \\
6.50 & 0.45 & $30.1(31)$ & $60.5(63)$ \\
6.75 & 0.65 & $20.8(21)$ & $41.9(43)$ \\
7.00 & 0.91 & $14.9(15)$ & $29.9(31)$ \\
7.25 & 1.27 & $10.7(11)$ & $21.5(22)$ \\
7.50 & 1.72 & $7.9(8)$ & $15.8(16)$ \\
7.75 & 2.22 & $6.1(6)$ & $12.3(12)$ \\
8.00 & 2.56 & $5.3(5)$ & $12.6(11)$ \\
8.25 & 2.22 & $6.1(6)$ & $16.6(12)$ \\
8.50 & 1.64 & $8.2(8)$ & $26.7(27)$ \\
8.75 & 1.02 & $13.3(13)$ & $43.2(44)$ \\
9.00 & 0.63 & $21.5(22)$ & $64.9(67)$ \\
\hline
\end{tabular}

${ }^{a}$ Conditions: $\tau_{d}=1.5 \mathrm{~s} ; \tau_{m}=0.5 \mathrm{~s}, \tau_{s}=1 \mathrm{~s} ; \tau_{e}=3 \mathrm{~s}$. ${ }^{\mathrm{b}}$ Conditions: $\tau_{d}=1.5 \mathrm{~s} ; \tau_{m}=0.5 \mathrm{~s}$, $\tau_{\mathrm{s}}=1 \mathrm{~s} ; \tau_{e}=0 \mathrm{~s}$.

an ISFET, respectively, calculated on the basis of the control criteria given in the previous section. A value of $\omega$ can be calculated for the phase margin $\phi=-130^{\circ}$ from Eqn. (11). This value is substituted in Eqn. (10) for $|H(j \omega)|$ $=1$. From this calculation the maximum value of $K_{2}$ is found which obeys the phase margin. In the same way, a value of $K_{2}$ can be calculated to obey the gain margin $\left(\phi=-180^{\circ}\right.$ and $\left.|H(j \omega)|=0.5\right)$. In order to fulfil both criteria, the smallest value of $K_{2}$ is taken in Table 1 .

From the table it can be seen that the steepest part of the titration curve is between $\mathrm{pH} 7.5$ and 8 . This is due to the fact that the second dissociation of sulphuric acid is weak $\left(K_{\mathrm{a}}=1.2 \times 10^{-2}\right)$. Calculation shows that the equivalence point is at approximately $\mathrm{pH} \mathrm{7.8.} \mathrm{The} \mathrm{slope} \mathrm{of} \mathrm{the} \mathrm{titration} \mathrm{curve}$ ( $K_{1}$ values) is influenced by the buffering properties of dissolved impurities and is thus somewhat smaller than would be expected for a pure sodium sulphate solution. Dissolution of carbon dioxide causes the $K_{1}$ values to change in time. After some hours in use, however, the titration curve tends to stabilize resulting in the values given in Table 1.

The table is stored on a floppy disk and used in the desired control programs for optimal $\mathrm{pH}$ control. Note that the fast response of an ISFET (assumed $\tau_{e}=0 \mathrm{~s}$ ) results in a larger tolerable value of $K_{2}$ which makes the system as a whole faster and more stable. 


\section{RESULTS}

The $\mathrm{pH}$ control system was tested with a stepwise sequence of desired $\mathrm{pH}$ values for any desired span of time. The sampling time was set to $1 \mathrm{~s}$ and the coulostat output current to $0.1 \mu \mathrm{mol} \mathrm{s} \mathrm{s}^{-1}$ or $9.65 \mathrm{~mA}$. This means that the microprocessor has to calculate the sign and the duration of the fixed coulostat output current after each measuring sample of the actual $\mathrm{pH}$, in relation to the corresponding system parameters, from the stored table and at the desired $\mathrm{pH}$ value. The calculated pulse duration is also given in Table 1 for a minimum $\Delta \mathrm{pH}_{\min }=0.01$. The duration is $x$ times this value for a $\Delta \mathrm{pH}=x$ $\Delta \mathrm{pH}_{\text {min }}$. The calculated time is entered in a programmable clock circuit which controls the coulostat. After each sample a new calculation is carried out and the clock is reprogrammed. This means that the effective duration of a current pulse is limited to approximately one second. If the calculated time exceeds this value (large value of $\Delta \mathrm{pH}=\mathrm{pH}_{\text {desired }}-\mathrm{pH}_{\mathrm{actual}}$ ), the speed of the system becomes limited by the fixed output current from the coulostat, which determines the slope of the titration curve. A comparison between the system response times when a glass electrode or an ISFET is used as the $\mathrm{pH}$ sensor is therefore useful only for those cases where no limitation is imposed by the coulostat (pulse width $\tau$ (Fig. 4) $<1$ s).

Figure 5 shows the results of a stepwise sequence in $\mathrm{pH}$ when a glass electrode was used as the pH sensor (lower curve), and when an ISFET was used (middle curve). The small fluctuations of $\Delta \mathrm{pH}( \pm 0.01)$ are due to the limited resolution of the $A / D$ converter. It can be seen that the use of an ISFET is preferable with regard to the response time, in agreement with the calculated larger values of $K_{2}$ as shown in Table 1. The upper curve of Fig. 5 shows the instability of the system when a glass electrode is used and the value of $K_{2}$ taken is twice the permitted value.

The control system was developed for the elimination of interferences, e.g., from dissolution of carbon dioxide. To test the effectiveness of the system quantitatively, $0.03 \mathrm{ml}$ of $0.01 \mathrm{M} \mathrm{HCl}$ was injected at around $\mathrm{pH} 7$, the most sensitive pH area. The control action is shown in Fig. 6 for a glass elec-

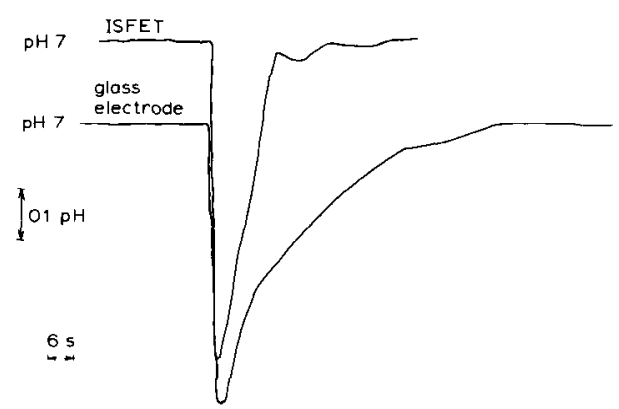

Fig. 6. Recovery of the $\mathrm{pH}$ after injection of $0.03 \mathrm{ml}$ of $0.01 \mathrm{M} \mathrm{HCl}$. The upper curve is for the ISFET with $K_{2}=21.5 \rightarrow 41.9 \rightarrow 21.5$; the lower curve is for the glass electrode with $K_{2}=10.7 \rightarrow 20.8 \rightarrow 10.7$. 
trode or an ISFET as the $\mathrm{pH}$ sensor. Because of the larger value of $K_{2}$ in the latter case, the effect of the disturbance is much shorter which is in complete agreement with the theoretical expectation as described by Eqn. (8).

\section{Conclusions}

It is shown that a system can be designed and made to control the $\mathrm{pH}$ of a certain volume of unbuffered solution. The accuracy and the response time of the system depend on the ion sensor which is used to measure the actual $\mathrm{pH}$ of the solution. The faster the response time of this sensor, the larger the open-loop amplification of the system that can be chosen, and the more stable the system will be. Therefore an ISFET as pH sensor is preferable to a glass electrode. It will be clear that other programs can also be implemented in the system. For example, to obtain a linear increase or decrease of $\mathrm{pH}$, the desired $\mathrm{pH}$ value is simply programmed as a function of time. The maximum slope of a linear $\mathrm{pH}$ change is again a function of the response time of the ion sensor.

The authors thank Dr. M. Bos from the Department of Chemical Technology for his help and useful suggestions.

\section{REFERENCES}

1 F. H. Raven, Automatic Control Engineering, 3rd edn., McGraw-Hill, New York, 1978, Ch. 11, 12.

2 L. J. Bousse, P. Bergveld and W. E. van der Linden, Scientific Session on Electrochemical Detection in Flow Analysis, October, 1982, Matrafüred, Hungary. 